30TH INTERNATIONAL COSMIC RAY CONFERENCE

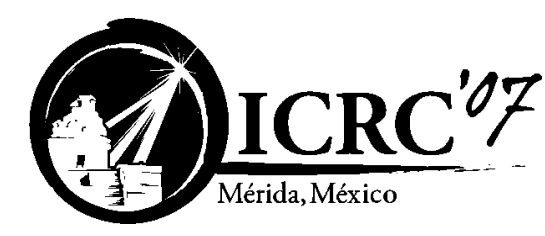

\title{
Diffuse Gamma-Rays Produced in Cosmic-Ray Interactions and the TeV-band Spec- trum of RX J1713.7-3946
}

\author{
C.-Y. Huang, M. Pohl, S.-E. PARK And C. D. Daniels \\ Department of Physics and Astronomy, Iowa State University, Ames, IA 50011 \\ huangc@iastate.edu
}

\begin{abstract}
We employ the Monte Carlo particle collision code DPMJET3.04 to determine the multiplicity spectra of various secondary particles (in addition to $\pi^{0}$ 's) with $\gamma$ 's as the final decay state, that are produced in cosmic-ray ( $p$ 's and $\alpha$ 's) interactions with the interstellar medium. We derive an easy-touse $\gamma$-ray production matrix for cosmic rays with energies up to about $10 \mathrm{PeV}$. This $\gamma$-ray production matrix is applied to the $\mathrm{GeV}$ excess in diffuse Galactic $\gamma$-rays observed by EGRET, and we conclude the non- $\pi^{0}$ decay components are insufficient to explain the GeV excess, although they have contributed a different spectrum from the $\pi^{0}$-decay component. We also test the hypothesis that the TeV-band $\gamma$-ray emission of the shell-type SNR RX J1713.7-3946 observed with HESS is caused by hadronic cosmic rays which are accelerated by a cosmic-ray modified shock. By the $\chi^{2}$ statistics, we find a continuously softening spectrum is strongly preferred, in contrast to expectations. A hardening spectrum has about $1 \%$ probability to explain the HESS data, but then only if a hard cutoff at $50-100 \mathrm{TeV}$ is imposed on the particle spectrum.
\end{abstract}

\section{The $\gamma$-Ray Production Matrix}

It is generally believed that diffuse galactic $\gamma$-rays are a good probe of the production sites and also the propagation of accelerated charged particles in the Galactic plane (see [3] and references therein). Exact knowledge of the $\gamma$-ray source spectrum resulting from hadronic interactions is of prime importance for a proper physical interpretation for the diffuse $\gamma$-rays. The production of neutral pions and their subsequent decay to $\gamma$-rays is thought to be the principal mechanism for the $\gamma$-ray hadronic component in cosmic ray interactions, and a number of parameterizations have been developed to describe $\gamma$-ray production in $p p$-collision [5, 8]. On the other hand, in addition to the neutral pion production, the direct $\gamma$-ray production in cosmicray interaction was found important [7], as was the production of $\left(\Sigma^{ \pm}, \Sigma^{0}\right),\left(K^{ \pm}, K^{0}\right)$, and $\eta$ particles, all of which eventually decay into $\gamma$-rays [8]. Also, the helium nuclei in cosmic rays and the interstellar medium are expected to contribute about $20-30 \%$ to the secondary production in cosmic-ray interactions, for which the multiplicity spectra may be different. In a hadronic interaction, the multi- plicity of non- $\pi^{0}$ secondaries with $\gamma$-rays as the final decay states is about $50 \%$ of the $\pi^{0}$ multiplicity. These non- $\pi^{0}$ secondaries in hadronic interactions might present a different $\gamma$-ray spectral profile.

This work presents a careful study of the $\gamma$-ray production in cosmic-ray (both $p$ and $\alpha$ ) interactions, by accounting for all decay processes including the direct production. For that purpose we employ the event generator DPMJET-III [10] to simulate secondary productions in both p-generated and He-generated interactions. We include all relevant secondary particles with $\gamma$-rays as the final decay products. For the composition of the ISM, we assume $90 \%$ protons, $10 \%$ helium nuclei, $0.02 \%$ carbon, and $0.04 \%$ oxygen. Around the energy of $\pi^{0}$ production threshold, where DPMJET appears unreliable, we apply a parametric model [5], that includes the resonance production for the $\pi$ production. We thus derive a $\gamma$-ray production matrix for cosmic rays with energies up to about $10 \mathrm{PeV}$ that can be easily used to interpret the spectra of cosmic $\gamma$-ray sources.

We consider all the decay channels and their decay fractions published by the Particle Data Group to account for all secondaries (resonances included) 
calculated by DPMJET and the parametric method. The calculation shows the non- $\pi^{0}$ resources in hadronic interactions have contributed about $20 \%$ of the total $\gamma$-ray photons, mostly from directly produced $\gamma$-rays and decays of $\eta, K_{L}^{0}$ and $K_{S}^{0}$ [7].

In the cosmic-ray interactions, we calculate the $\gamma$ ray spectrum density contributed by decays of unstable secondary particles

$\frac{Q_{\gamma}\left(E_{\gamma}\right)}{n_{I S M}}=\sum_{k} \int d E N_{C R}(E) c \beta \sigma(E) \frac{d n_{k, \gamma}}{d E_{\gamma}}$

where $\frac{d n_{k, \gamma}}{d E_{\gamma}}=\frac{d n_{k, \gamma}}{d E_{\gamma}}\left(E_{\gamma}, E\right)$ is the $\gamma$-ray decay spectrum from secondary species $k$. Eq. (1) can be re-written into

$$
\begin{aligned}
& \frac{Q_{\gamma}\left(E_{i}\right)}{n_{I S M}} \\
= & \sum_{j} \Delta E_{j} N_{C R}\left(E_{j}\right) c \beta_{j} \sigma\left(E_{j}\right) \sum_{k} \frac{d n_{k, \gamma}}{d E_{\gamma}} \\
= & \sum_{j} \Delta E_{j} N_{C R}\left(E_{j}\right) c \beta_{j} \sigma_{j} \mathcal{M}_{i j}
\end{aligned}
$$

thus reducing this problem to a matrix operation with the $\gamma$-ray production matrix $\mathcal{M}_{i j}$ for which, each element $\mathcal{M}_{i j}=\sum_{k} \frac{d n_{k, \gamma}}{d E_{\gamma}}$ shows the value of the resultant particle energy spectrum $\left.\frac{d n}{d E}\right|_{E_{\gamma}=E_{i}, E_{C R}=E_{j}}$, with $j$ being the index for the generating cosmic-ray particle ( $p$ or $\alpha$ ) and $i$ being the index indicating the $\gamma$-ray energy. The energy binnings $E_{i}$ and $E_{j}$ are defined with good resolutions [7].

We then use the $\gamma$-ray production matrix to analyze the observed spectra of diffuse Galactic emission and of the shell-type SNR RX J1713.7-3946.

\section{The GeV Excess}

With the $\gamma$-ray production matrix, we calculate the diffuse $\gamma$-ray spectrum generated by the observed cosmic-ray spectrum [9]. Fig. 1 shows the observed $\mathrm{GeV}$-band $\gamma$-ray emission from the inner Galaxy [4] in comparison with the contributions from $\pi^{0}$ decay as well as bremsstrahlung emission describe by a power-law spectrum $\Phi_{\mathrm{B}}(E)$ :

$$
\begin{aligned}
\Phi_{\mathrm{B}}(E) & \simeq 1.3 \times 10^{-8} \frac{\omega_{e}}{0.1 \mathrm{eV} / \mathrm{cm}^{3}} \cdot \frac{N_{I S M}}{10^{22} \mathrm{~cm}^{-2}} \\
& \times\left(\frac{E}{100 \mathrm{MeV}}\right)^{2.0-\Gamma_{e}} \frac{\mathrm{erg}}{\mathrm{cm}^{2} \mathrm{sec} \mathrm{sr}}
\end{aligned}
$$

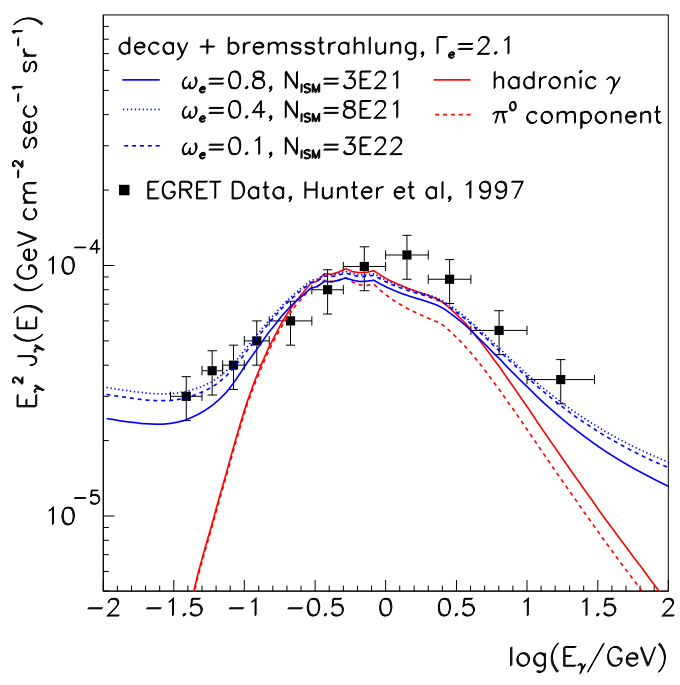

Figure 1: Diffuse $\gamma$-ray spectrum at $\mathrm{GeV}$ range, shown in comparison with data for the inner part of Galaxy at $315^{0} \leq l \leq 345^{\circ}$ and $|b| \leq 5^{0}[4]$. See text for discussions.

with power-law spectral index $\Gamma_{e}=2.1$, the electron energy density $\omega_{e}=0.1,0.4,0.8 \mathrm{eV} / \mathrm{cm}^{3}$, and gas column density $N_{I S M}=3 \cdot 10^{22}, 8$. $10^{21}, 3 \cdot 10^{21} \mathrm{~cm}^{-2}$ respectively. The generating cosmic-rays are assumed with an energy density $\rho_{E}=0.75 \mathrm{eV} / \mathrm{cm}^{3}$. Models based on the locally observed cosmic-ray spectra generally predict a softer spectrum for the leptonic components, even after accounting for inverse Compton emission [4], so we may in fact overestimate the GeV-band intensity of the leptonic contribution. Nevertheless, it is clearly seen in this figure, that in the total intensity an over-shooting around $E_{\gamma} \simeq 300-600 \mathrm{MeV}$ appears in the modelled $\gamma$ ray energy distribution, whereas a deficit is present above $1 \mathrm{GeV}$. The observed spectrum of diffuse emission is always harder than the model spectrum, and we therefore conclude that an inaccurate description of hadronic $\gamma$-rays is ruled out as the origin of the $\mathrm{GeV}$ excess.

\section{TeV-band Emission from RX J1713.7-3946}

For the TeV-band $\gamma$-ray spectrum of the shell-type SNR J1713.7-3946 observed by the HESS collaboration [6], we use the $\gamma$-ray production matrix to test cosmic-ray acceleration models $[1,2]$, which predict a continuous hardening of the cosmic-ray 


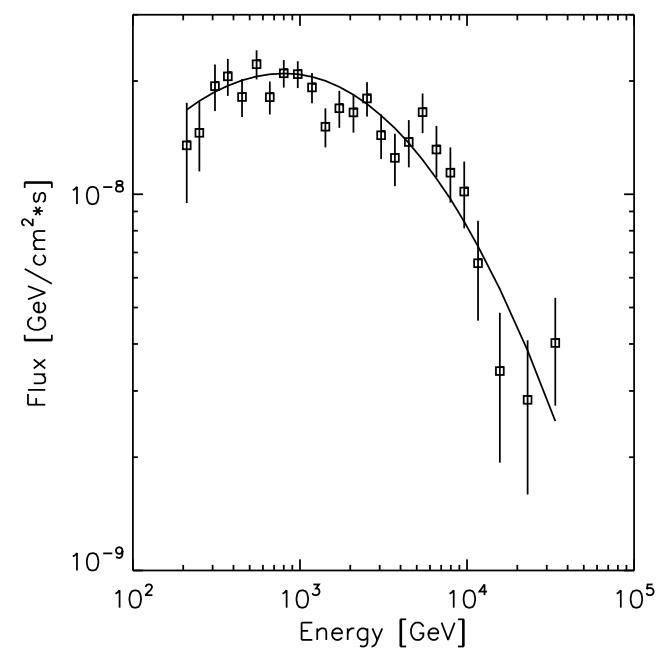

Figure 2: The TeV-band $\gamma$-ray spectrum observed from RX J1713.7-3946 with HESS [6], shown in comparison with the best-fit model of hadronic $\gamma$ ray production of Eq. (4).

spectrum up to a high-energy cutoff. We therefore parametrize the spectrum of accelerated hadrons as

$N(E)=N_{0}\left(\frac{E}{E_{0}}\right)^{-s+\sigma \ln \frac{E}{E_{0}}} \Theta\left[E_{\max }-E\right]$

where $\Theta$ is the step function and $E_{0}=15 \mathrm{TeV}$ is a normalization chosen to render variations in the power-law index $s$ statistically independent from the choice of spectral curvature, $\sigma$. The cutoff energy, $E_{\max }$, is a free parameter. The normalization $N_{0}$ is obtained by normalizing both the data and the model to the value at $0.97 \mathrm{TeV}$. By the $\chi^{2}$ statistics, we obtain the best-fitting values and the confidence ranges of the three parameters, $E_{\max }$, $s$, and $\sigma$, given values as $s=2.13, \sigma=-0.25$, and $E_{\max } \geq 200 \mathrm{TeV}$, i.e., no cutoff. The best fit, shown in Fig. 2, involves a continuous softening and is thus not commensurate with expectations based on acceleration at a cosmic-ray modified shock $[1,2]$. Note the need for data in the energy range between $1 \mathrm{GeV}$ and $200 \mathrm{GeV}$ that may be provided by GLAST in the near future. Fig. 3 and Fig. 4 shows the confidence ranges of the parameters in Eq. (4), for $\sigma$ vs. $s, \sigma$ vs. $E_{\max }$ and $s$ vs. $E_{\max }$ respectively, with confidence levels of 1,2 and 3 sigma. The contour for $E_{\max }$ are open toward higher energies, indicating that a cut-off is not statistically required. The analysis shows that

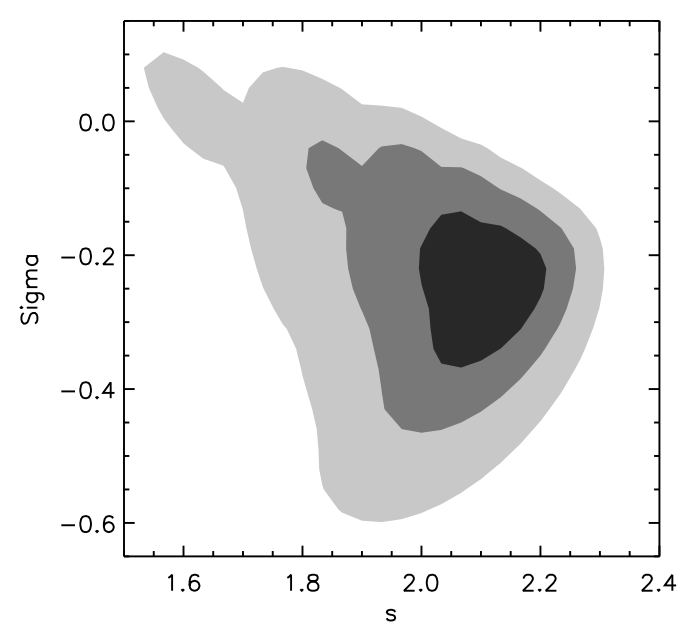

Figure 3: The confidence regions for the spectral curvature $\sigma$ and the spectral index $s$. The shaded areas correspond to probabilities 68\% (1 sigma), 95\% (2 sigma) and 99.7\% (3 sigma).

a very high $E_{\max }$ is possible, but requires a negative spectral curvature, i.e., $\sigma<0$, with confidence more than $95 \%$, in contrast to the expectation of standard cosmic-ray modified shock models.

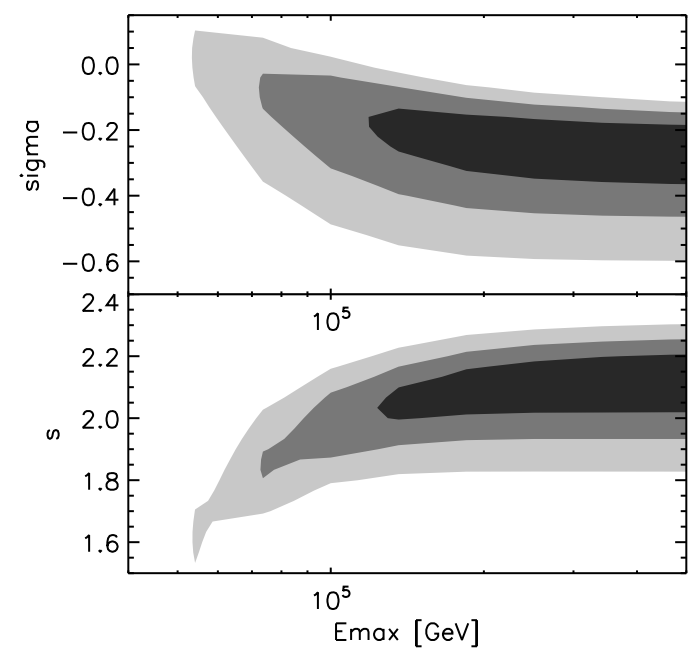

Figure 4: Confidence contours as in Fig. 3.

Note the step function term $\Theta\left(E_{\max }-E\right)$ in Eq. (4) can be replaced by an exponential term $\exp \left(-E / E_{\max }\right)$. Fig. 5 shows the confidence level of $E_{\max }$ vs. $s$ for the new best-fit parametrization. The contour shows a minor deviation from original confidence contours in Fig. 4, however, the conclusion is not altered. 


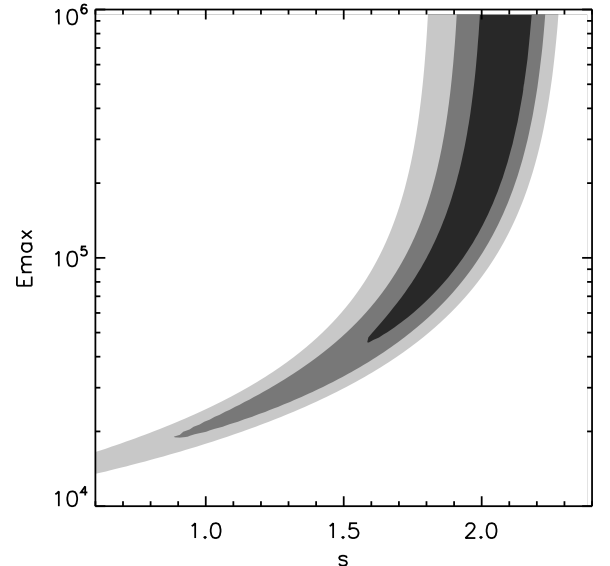

Figure 5: Confidence contour as in Fig. 3 but for an exponential term $\exp \left(-E / E_{\max }\right)$ in Eq. (4) instead of the step function.

\section{Conclusions}

We have considered a full picture of the hadronic $\gamma$-rays in cosmic-ray interactions and introduced an easy-to-use $\gamma$-ray production matrix which can be used for arbitrary cosmic-ray spectrum. The matrices are available for download at website http://cherenkov.physics.iastate.edu/gamma-prod. We apply the production matrix to calculate the $\gamma$-ray $\mathrm{GeV}$ excess and also the $\mathrm{TeV}$-band spectrum of SNR RX J1713.7-3946. We conclude that 1) the modifications in the GeV-band $\gamma$-ray emission of hadronic origin are insufficient to explain the $\mathrm{GeV}$ excess in diffuse galactic $\gamma$-rays; 2) a soft cut-off at about $100 \mathrm{TeV}$ is statistically required in the particle spectrum if the TeV-band spectrum of RX J1713.7-3946 as observed with HESS is caused by cosmic-ray nucleons; 3) no evidence for efficient nucleon acceleration to energies near the knee in the cosmic-ray spectrum, nor evidence of the spectral curvature and hardness predicted by standard models of cosmic-ray modified shock acceleration. We emphasize the need for GLAST data to better constrain the $\gamma$-ray spectrum below $100 \mathrm{GeV}$.

In addition to $\gamma$-ray production matrix, we have also produced the production matrices of all particles that are stable on a time scale relevant for cosmic-ray propagation. The leptonic production by the same approach will be discussed in an independent article.

\section{Acknowledgments}

Grant support from NASA with award No. NAG513559 is gratefully acknowledged.

\section{References}

[1] E. Amato and P. Blasi. Non-linear particle acceleration at non-relativistic shock waves in the presence of self-generated turbulence. MNRAS, 371:1251-1258, 2006.

[2] E. G. Berezhko and D. C. Ellison. A Simple Model of Nonlinear Diffusive Shock Acceleration. APJ, 526:385-399, 1999.

[3] A.D. Erlykin, M. Lipski, and A.W. Wolfendale. High energy cosmic ray spectroscopy. IV. The evidence from direct observations at lower energies and directional anisotropies. Astropart. Phys, 8:283-292, 1998.

[4] S. D. Hunter et al. EGRET Observations of the Diffuse Gamma-Ray Emission from the Galactic Plane. APJ, 481:205-240, 1997.

[5] T. Kamae et al. Parameterization of $\gamma, e^{ \pm}$ and Neutrino Spectra Produced by p-p Interaction in Astronomical Environments. APJ, 647:692-708, 2006.

[6] F. A. Aharonian et al. The HESS Collaboration. A detailed spectral and morphological study of the gamma-ray supernova remnant RX J1713.7-3946 with HESS. AA, 449:223242, 2006.

[7] C.-Y. Huang, S.-E. Park, M. Pohl, and C.D. Daniels. Gamma-rays produced in cosmicray interactions and the TeV-band spectrum of RX J1713.7-3946. Astropart. Phys, 27:429-439, 2007.

[8] S. R. Kelner, F. A. Aharonian, and V. V. Bugayov. Energy spectra of gamma rays, electrons, and neutrinos produced at protonproton interactions in the very high energy regime. PRD, 74:034018-034033, 2006.

[9] M. Mori. The Galactic Diffuse Gamma-Ray Spectrum from Cosmic-Ray Proton Interactions. APJ, 478:225-232, 1997.

[10] S. Roesler, R. Engel, and J. Ranft. Advanced Monte Carlo for Radiation Physics. In Particle Transport Simulation and Applications (MC 2000), 2000. 